\title{
CARDIAC MUSCLE
}

\section{Its Ultrastructure in the Finch and Hummingbird with Special Reference to the Sarcoplasmic Reticulum}

\author{
PAUL H. JEWETT, J. R. SOMMER, and E. A. JOHNSON
}

From the Departments of Pediatrics, Pathology, and Physiology, Duke University Medical Center, and the Veterans Administration Hospital, Durham, North Carolina 27706

\begin{abstract}
A BSTRA C T
Cardiac muscle fibers of the hummingbird and finch have no transverse tubules and are smaller in diameter than those of mammalian hearts. The fibers are connected by intercalated discs which are composed of desmosomes and $f$. adherentes; small nexuses are often interspersed. As in cardiac muscle of several other animals, the junctional SR of the couplings is highly structured in these two birds but, in addition, and after having lost sarcolemmal contact, the junctional SR continues beyond the coupling to extend deep into the interior of the cells and to form belts around the Z-I regions of the sarcomeres. This portion of the sarcoplasmic reticulum, which we have named "extended junctional SR," and which is so prominent and invariant a feature of cardiac cells of hummingbirds and finches, has not been observed in chicken cardiac cells. The morphological differences between these species of birds may be related to respective differences in heart rates characteristic for these birds.
\end{abstract}

\section{INTRODUCTION}

Comparison of the fiber structure of hearts of different animals can provide a great deal of insight into the relationships between the structure of cardiac muscle and its function; during phylogenesis and ontogenesis essential features of structure, and of function, survive.

We have chosen to select the hearts of the hummingbird and of the finch for a comparative look because of the very fast heart rates which distinguish these two species from the previously studied chicken (1). We have further chosen to set aside the myofilaments, for it appeared to be more rewarding, at the moment, to say something about forms of cell apposition, and about the ultrastructure and topography of the sarcoplasmic reticulum.

The ultrastructure of the heart of the hummingbird has been investigated before, but these studies were primarily concerned with a description of mitochondrial morphology (2). Some features of the anatomy of heart muscle from a variety of other birds have previously been reported (3-9). The present inquiry adds the description of a discrete anatomical feature in cardiac muscle of the hummingbird and finch, which consists of a strikingly prominent morphological differentiation of portions of the sarcoplasmic reticulum and which, because of its extent and because of its specific intracellular location, provokes intriguing speculations as to its possible functional role.

\section{Definitions, Criteria, and Dimensions}

For additional terminology the reader is referred to previous publications (10-14). Our find- 
ings in the finch and hummingbird have necessitated the subdivision of the sarcoplasmic reticulum (SR) into the following components, although all are continuous with one another. The subdivisions are (a) free, (b) junctional, and $(c)$ extended junctional, $\mathrm{SR}$. The free SR is that portion of the SR which is composed of a network of tubules (20-80 $\mathrm{m} \mu$ wide) that, electron optically, have usually a clear lumen and that surround the $\mathrm{A}$ bands of the sarcomeres; it is that part of the SR that does not have structural features of the junctional, nor of the extended junctional, SR. The junctional SR (11) refers to that portion of the SR which contains electronopaque granules (junctional granules), and that forms couplings (10) with the sarcolemma, either at the periphery of the cell (peripheral couplings; mammals and birds), or at transverse tubules, i.e., in the interior of the cell (interior couplings; mammals) (11, 12). Sandwiched between the junctional SR and the sarcolemma, as part of the couplings, there are seen periodic densities (junctional processes). In the finch and hummingbird a portion of the SR is intercalated between the junctional SR and the free SR that has the morphology of junctional SR (i.e. SR tubule, junctional granules, junctional processes), but that is not in contact with the sarcolemma. We have named this SR portion, tentatively, "extended junctional SR" because, except for the absence of actual contact with sarcolemma, its morphology appears identical to that of the junctional SR of couplings.

The nexuses noted in the two birds under study are gap junctions. We have avoided the terms "macula" and "fascia occludens" in this paper, and refer to all of these structures simply as nexuses (15), because that indifferent term names a structurally discrete entity without qualification; "macula" and "fascia" signify, yet do not specify, different sizes of these junctional complexes. The use of the word "occludens" has become inappropriate since a gap was discovered between the unit membranes of the nexus (16).

$M$ fibers (working myocardium) only are described in this report. Although we have seen apparent $\mathbf{P}$ fibers with the light microscope (cf. 17), there are surprisingly few of them as compared with the chicken.

All magnifications are reasonable approximations within the limits of a routinely calibrated electron microscope, and within the limits of routine photographic processing.

\section{MATERIALS AND METHODS}

\section{Animals}

Eight adult zebra finches (Taeniopygia castanotis) of either sex, one adult female hummingbird (rufous tailed), and one adult female chicken (Leghorn) were anesthesized with ether. The beating hearts were removed, opened, and immersed in fixative for $2-4 \mathrm{hr}$. Small samples of ventricular and atrial subendocardial myocardium, not more than $0.2 \mathrm{~mm}$ thick, were dissected out about 30 min after immersion in the fixative, and then left in the fixative for the remainder of the time.

\section{Fixatives}

Either $4 \%$ glutaraldehyde in 0.2 m cacodylate buffer with $5 \mathrm{~mm}$ calcium chloride, or Karnovsky's fixative (18) was used at room temperature and at $\mathrm{pH}$ 7.2-7.4. Tissue samples were rinsed for $30 \mathrm{~min}$ in either distilled water or $0.2 \mathrm{~m}$ cacodylate buffer, postfixed in freshly prepared $2 \%$ osmium tetroxide in Veronal-acetate buffer (19) for $2 \mathrm{hr}$. dehydrated through graded alcohols, and embedded in either Maraglas or Epon 812. Some tissues were contrasted with uranyl acetate in $50 \%$ ethanol or water en bloc for $60 \mathrm{~min}$ after osmification. All specimens were stained on the grid with lead citrate.

\section{Special Studies}

Finch and young chicken (3-5 days old) hearts were injected with $100 \mathrm{mg}$ of horseradish peroxidase in $3 \mathrm{ml}$ of Ringer solution (20). After administration of ether anesthesia the thorax was opened, the aorta was clamped, and the peroxidase solution was injected by hand syringe through a 22 gauge needle into the right and left ventricular cavities with sufficient force to moderately distend the ventricle. A heart of an adult hen was also injected with peroxidase in a similar manner (500 $\mathrm{mg}$ in $15 \mathrm{ml}$ of Ringer solution). After injection of the exogenous peroxidase, the hearts were quickly removed, opened, and immersed for 4 $\mathrm{hr}$ in $4 \%$ glutaraldehyde in cacodylate buffer as above (see Fixatives). Samples about $0.2 \mathrm{~mm}$ thick were then dissected out, rinsed briefly in buffer, and exposed for 15-30 min to a solution containing $20 \mathrm{ml}$ Tris buffer, $10 \mathrm{ml} 3-3$-diaminobenzidine tetrahydrochloride, and $1 \mathrm{ml} 1 \%$ hydrogen peroxide. These samples were then postfixed in $2 \%$ osmium tetroxide in Veronal-acetate buffer for $11 / 2 \mathrm{hr}$. dehydrated through graded alcohols, and embedded in Epon 812 without further contrast enhancement, and thin sections were cut for electron microscopy.

For measurement of fiber diameters, similar multiple small ( $0.2 \mathrm{~mm}$ thick) individual ventricular myocardial samples from these hearts were separately 


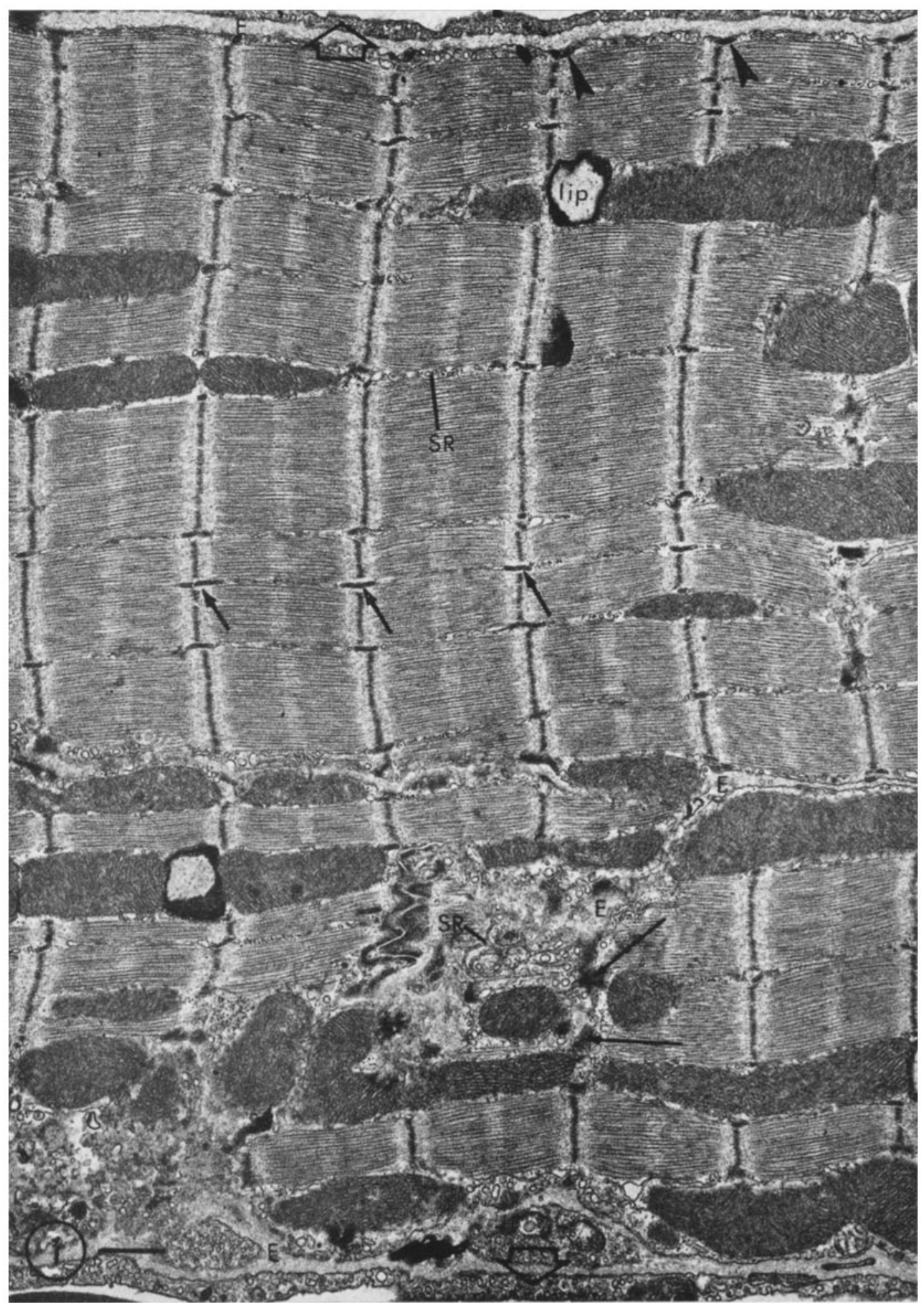

FIGURE 1 Hummingbird. Ventricular fibers contain numerous profiles of extended junctional SR (arrows) in the interior of the fibers at the $Z$ lines and away from contact with the sarcolemma. Transverse tubules are absent. Junctional SR forms peripheral couplings at $Z$ lines (arrowheads). Free SR (SR) fills the interfibrillary spaces around A bands. Many mitochondria and lipofuscin deposits (lip) are present. Extracellular space $(E)$. Open arrows mark endothelial cells. Scale, $1 \mu$. $\times 11,000$. 


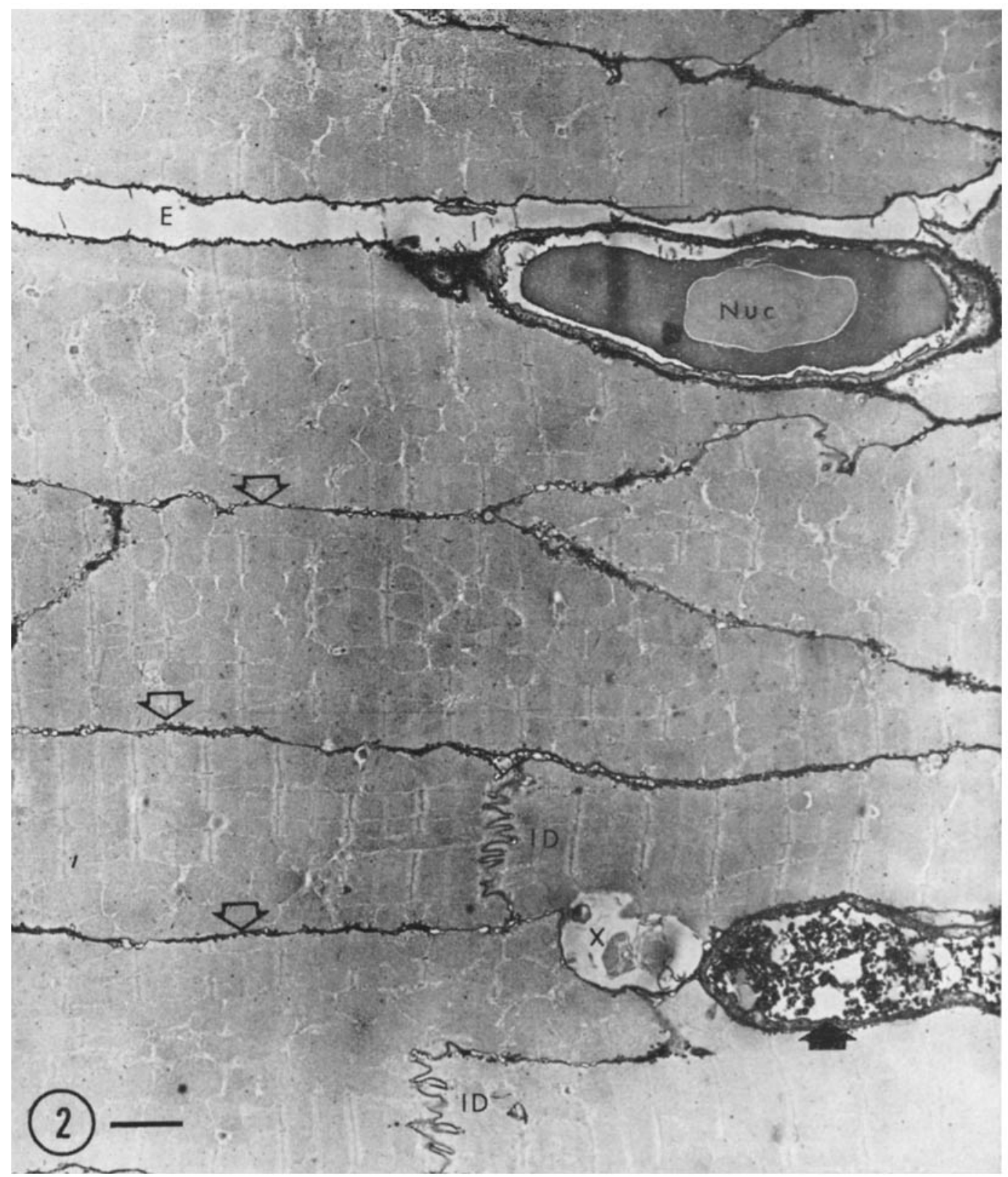

Figure 2 Finch, ventricular fibers. Exogenous peroxidase is seen in capillaries (large black arrow), in the wide $(E)$ and narrow (open arrows) extracellular spaces, and in the intercalated discs $(I D)$. The interior of the fibers is free of reaction product, demonstrating the absence of transverse tubules. Nucleated red blood cell, $N u c$; fixation artifacts, $X$. Scale, $2 \mu . \times 5000$.

embedded in Epon 812, and $1 \mu$ thick cross-sections were cut, mounted on glass slides, and photographed with a Leitz Orthomat (phase contrast) (E. Leitz Inc., Rockleigh, N.J.). A Unitron slide micrometer was used as a calibration standard (Budd Co., Instrument Div., Phoenixville, $\mathrm{Pa}$.). No more than one section from each ventricular sample was studied to preclude measuring any fiber more than once. 50 such fibers 
were measured from each bird, and the greatest cell diameter was recorded, without respect to its coincidence with the nuclear region of the cell. Calculations of the range and mean diameter of myofibers of finch, young chick, and adult hen were made. Areas of preparations where peroxidase did not completely fill the extracellular space were excluded from study, since fiber diameter measurements are subject to considerable error when all cell borders cannot be clearly distinguished, which is frequently the case in bird cardiac muscle because the individual cells often are separated by only extremely narrow intercellular spaces $(20-30 \mathrm{~m} \mu)$. Peroxidase, or more likely 3-3diaminobenzidine, only penetrated superficially into these preparations, and therefore, it was only from the superficial areas that fiber diameter measurements could be attempted.

\section{Sectioning and Microscopy}

Thin sections were cut on a Porter-Blum MT-1 or a Reichert OmU2 automatic ultramicrotome with diamond knives, suspended on collodion- and carboncoated grids, and viewed with either the RCA EMU$3 F(50 \mathrm{kv})$ or the AEI EM-6B (60 kv) electron microscope.

\section{RESULTS}

Cardiac muscle of the finch and hummingbird appeared similar. ${ }^{1}$ The myofibrils contained thick

${ }^{1}$ Subsequently we have had the opportunity to examine an adult canary heart and have confirmed and thin filaments forming $\mathrm{A}$ and I bands, but had no apparent $M$ lines. Mitochondria were numerous and showed complex cristae, though perhaps less marked than is seen in the canary (3). Individual fibers were surrounded by a typical sarcolemma (plasma-plus basement membrane) having numerous sarcolemmal vesicles and pits along its surface. The fibers were arranged in tightly packed bundles (Figs. 2, 3, 4, 11, 18), rather like those in the hearts of frogs and chickens, although of a somewhat larger over-all diameter $(10-30 \mu)$, with narrow (approximately 25-60 $\mathrm{m} \mu$ ) extracellular spaces. Wide $(1 \mu)$ extracellular spaces separating such bundles contained unmyelinated nerve fibers, capillaries, and collagen. Within the cells, lipid droplets, Golgi zones, lysosomes, lipofuscin granules, and rarely, portions of rough SR, were seen (Fig. 1). Transverse tubules were never observed, either in routinely stained sections (Fig. 1) or when extracellular spaces were marked with perioxidase (Fig. 2). The sarcoplasmic reticulum (SR) was found to form a profuse network extending across the $Z$ lines, often via extended junctional SR (Fig. 1). Many peripheral couplings were present (Figs. 1, 3 and 6). They

the presence of extended junctional SR in yet another fast-beating avian heart. Fig. 4 of reference 6 suggests that extended junctional SR is present also in the love bird.

FigURe 3 Finch, ventricular fibers near a blood vessel $(B V)$. Open arrow at top marks endothelial cell. Peripheral couplings at $C$. Dark arrow at top marks basement membrane along the sarcolemma. Profiles of extended junctional SR (TSR) extend into the fiber interior, forming a partial belt around myofibrils, and frequently envelop mitochondria $(M i)$. A clear space often seems to separate extended junctional SR from $Z$ substance ( $X$ in Fig. 5), although periodic connections seem to exist between TSR and $Z$ substance (large open arrow). Free SR, $S R$; A band, $A$; ZI region, $Z I$; junctional SR, $J S R$. Scale, 0.5 $\mu . \times 34,500$.

Frgure 4 Finch. A coupling $(C)$ is continuous with free SR at arrow. The close topographic relationship between free SR and mitochondrial $(\boldsymbol{M} i)$ membranes is seen at open arrow. Extracellular space, $E$. Scale, $0.125 \mu . \times 77,000$.

Frgure 5 Finch, extended junctional SR at high magnification. Junctional granules at open arrow bisect the lumen of the extended junctional SR and show periodic septate connections with the SR membranes. The periodic arrangement of the junctional processes gives the SR membranes a scalloped appearance (arrow heads). Arrows point to linear densities frequently seen crossing the clear space $(X)$ between extended junctional SR tubules and the $Z$ substance. Scale, $0.1 \mu . \times 150,000$.

FIGURe 6 Finch. Ventricular fibers in transverse section, demonstrating the extended junctional SR $(T S R)$ at the Z-I $(Z I)$ region of the sarcomere are visible while only free SR $(S R)$ is seen in A-band regions $(A)$. A nerve cell $(N)$ and membranous debris $(X)$ in the extracellular space $(E)$ are also visible. Coupling, $C$; junctional SR, JSR. Scale, $0.5 \mu . \times 20,000$. 

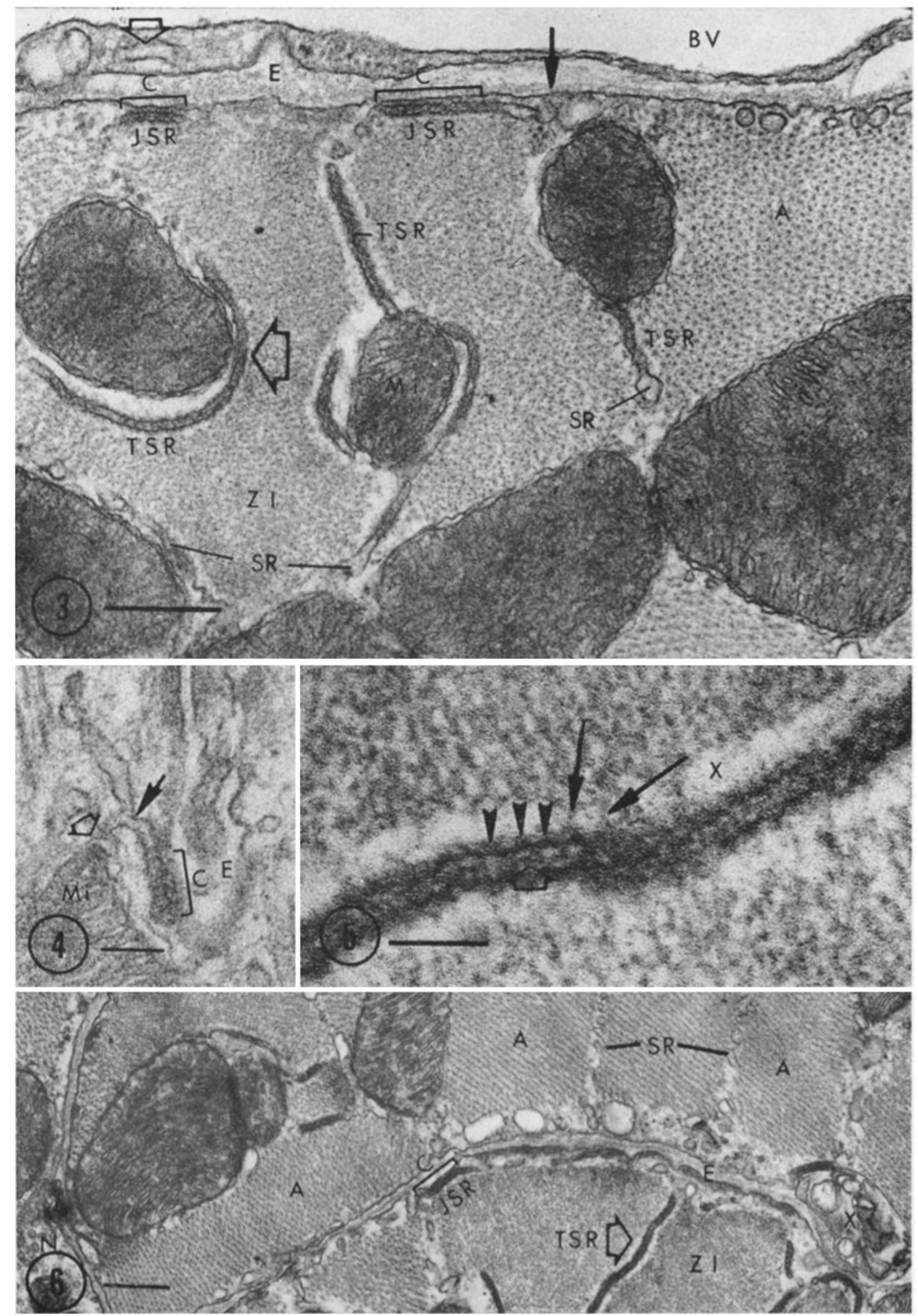

Paul H. Jewett, J. R. Sommer, and E. A. Johnson Cardiac Muscle 
were located predominately at $\mathbf{Z}$ lines and were continuous with both the free and extended junctional SR (Figs. 3, 4, 6, and 16). In the interior of the myofiber, and away from all contact with the sarcolemma, profiles of extended junctional SR were noted that appeared similar to and, in fact, often were identified as direct extensions of the junctional SR, the latter being an integral part of the peripheral couplings (Figs. 1, 3, and 6). This extended junctional SR was consistently found in the interfibrillar space at the $\mathrm{Z}-\mathrm{I}$ region of the sarcomere, while the free SR was confined to the spaces surrounding A-band regions (Fig. 6). In the lumen of the extended junctional SR, periodic densities were seen which appeared identical with the so-called junctional granules of junctional SR (11) and frequently formed quasi-continuous lines (Fig. 5); often, periodic septate connections were discernible between these electron-opaque lines and the inner aspects of the membranes forming the extended junctional SR. Processes were seen arising from the membranes of the extended junctional SR (Fig. 5). The similarity of these processes to the junctional processes of the junctional SR of couplings was striking. The processes occurred at regular, approximately 25$\mathrm{m} \mu$ intervals (Fig. 5), giving a scalloped appearance to the unit membranes of the extended junctional SR. Emanating from the processes and stretching toward the Z-line material, linear densities (Fig. 5) measuring approximately $10 \mathrm{~m} \mu$ in width were sometimes seen that suggested physical attachment of the extended junctional SR to the Z-line material. These linear densities at the external surface of the junctional processes alternated with respect to the position of the septate densities at the luminal surface of the extended junctional SR (Fig. 5). Often, the extended junctional SR appeared to surround a mitochondrion (Fig. 3), but no communication of the SR with the intermembranous space of mitochondria was demonstrated unequivocally (Fig. 4).

Grazing sections through the extended junctional SR revealed larger dimensions than those exposed by normal longitudinal or transverse sections (compare Fig. 7 with Figs. 3 and 6), indicating that the extended junctional SR forms a flat belt around the myofibrils at the $Z$ line. In some grazing sections, small, circular profiles were observed that suggested cross-sections of junctional processes (Fig. 8). These were similar to those re- ported by Kelly $(21,22)$ in terminal cisternae (junctional SR) of skeletal muscle. Dense bodies were found in both atrial and ventricular fibers (Figs. 9-11), but not so frequently as noted in the chicken (1). Electron-opaque material was found also in SR tubules (Fig. 10).

Typical intercalated discs were present between fibers (Figs. 4 and 5). Frequent small nexuses were noted in longitudinal portions (parallel to the axis of the myofibrils) of these discs (Figs. 13-16). Although areas of close membrane apposition (Fig. 16) occurred between cells laterally, no nexuses have thus far been found joining lateral cell borders away from the intercalated discs. Occasionally, relatively straight intercalated discs were seen (Fig. 16). The intercellular space in the intercalated discs was continuous with the extracellular space. The nexuses were gap junctions of typical dimension. Desmosomes were common.

Since nexuses were found to be numerous in the heart muscle of these birds, and had been found only rarely and with difficulty in developing chick heart muscle (1), an examination of adult hen ventricular muscle seemed appropriate, and indeed, small nexuses were noted between these fibers (Fig. 12). The diameter of $\mathrm{M}$ fibers (working fibers) of finch, hummingbird, and hen appeared roughly comparable, and noticeably larger than similar fibers from the developing chick. To confirm this impression, perioxidase was utilized as an extracellular tracer and cell borders appeared especially well outlined (Figs. 2 and 17-19).

Measurements of ventricular fiber diameters yielded for the finch (Fig. 17) a range of 4-10 $\mu$ with a mean of $6.9 \mu$. The corresponding measurements for the young chick (Fig. 19) were 2-6.7 $\mu$ with a mean of $4.3 \mu$ and for the adult hen (Fig. 18) $3.3-10 \mu$ with a mean of $6.1 \mu$.

The major findings of this investigation were (a) that nexuses are common in the hearts of the hummingbird, the finch, and the adult chicken, while in the developing chicken heart (embryonic and young chick) they are virtually absent $(1,14$, $23,24)$; (b) that a transverse tubular system is absent in the working muscle of hummingbird and finch hearts; and (c) that hearts of the hummingbird and finch differ significantly from the hearts of chickens, mammals and frogs, in that the former have a highly developed extended junctional SR but the latter do not. 


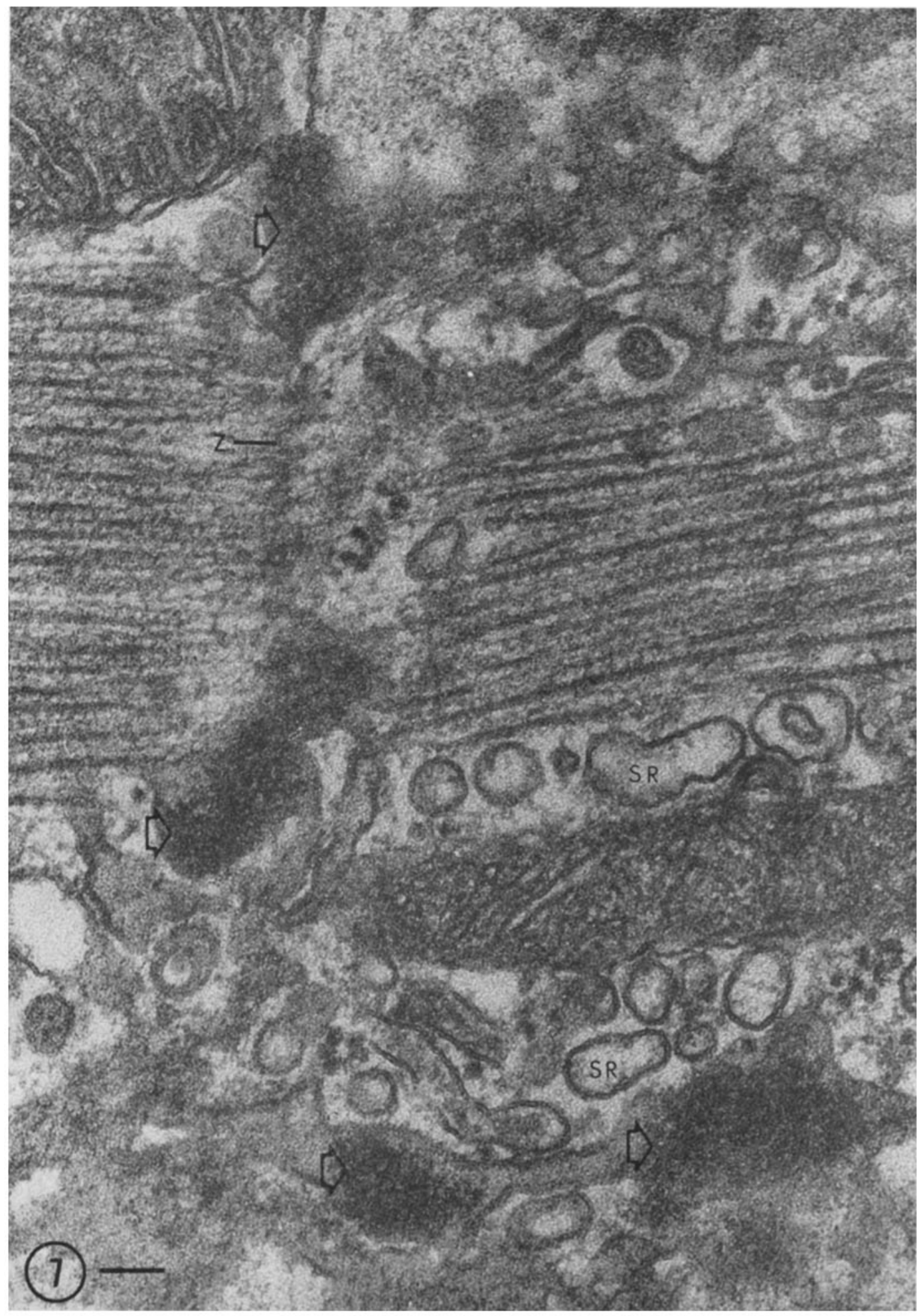

Figure 7 Hummingbird. Tangential section through extended junctional SR (open arrows) reveals a cribriform pattern, possibly a moire (see Discussion). The lumen of the extended junctional SR is larger in this tangential view than when sectioned normal to that plane, suggesting that the SR consists of a flattened tubule or sac surrounding the myofibril at the $Z$ line $(Z)$. Tubules of free SR (SR) are numerous. Scale, $0.1 \mu . \times 100,000$. 


\section{DISCUSSION}

\section{Nexuses}

Functionally, cardiac muscle cells behave as a syncytium, in that, electrically, they are resistively coupled. Ample proof of this is given by the fact that a resting membrane potential can be recorded in frog heart by the double-sucrose gap technique $(25,26)$, and that a steady electrical current injected into Inammalian cardiac cells causes a steady displacement in transmembrane potential in far distant cells (27-30).

The low resistance pathways between cardiac cells have been considered to be located in regions of close cell apposition and have been presumed to be associated with one form of such apposition, namely, the nexus. Although this notion is supported by the extensive and frequent occurrence of nexuses in cardiac muscle of mammals, it becomes open to question for the hearts of frogs, young chickens, and chicken embryos, since nexuses are rarely seen in these hearts (1). Whether we must relinquish the notion of the nexus as the sole location of low resistance pathways between cardiac cells depends on whether one can show that their size and frequency of occurrence are inadequate to perform such a function.

If the resistance of a unit area of nexus is relatively low compared with a unit area of sarcolemma, the size of a given nexus that is of physiological significance would depend on the input resistance of the cells at the site of the nexus. If the resistance of a nexus between two cells is comparable to, or smaller than, the input resistance of one or both cells, then, clearly, a sizable fraction of longitudinal current that might flow in one or the other cell at that point would change course and pass through the nexus to flow down the intracellu- lar pathway of the other. The input resistance, $R p$, (all other electrical factors, of importance remaining unchanged), depends on the geometry of the cell, so that, for example, for a cylindrical cell, $\mathrm{Rp}$ varies with the fiber radius, a, in the manner, $R p \propto$ $a^{-3 / 2}$. On the face of $i$, the seemingly smaller size of the majority of nexuses between cells of adult chicken and finch hearts compared with those between mammalian $\mathrm{V}$ fibers ( 1 ) could perhaps be accounted for by the relative difference in the diameter of the two kinds of fibers. This is compatible with the finding that nexuses of considerable size are present in the large Purkinje fibers of young chickens (23), and that small nexuses are found in the larger fibers of adult chickens $(24,14)$, hummingbirds, and finches, which are, again, larger than the cardiac fibers of young chickens. Indeed, when measured in the adult hen and finch, the average diameter of fibers in which nexuses are detectable is larger by at least $40 \%$ than the average diameter of fibers in the young chicken in which nexuses are virtually absent. On the other hand, in the case of ventricular fibers of the puppy and adult dog heart, where a similar difference in diameter occurred (unpublished observations), there was no striking difference in the frequency and size of nexuses.

Nevertheless, although the $M$ fibers of the frog (1) and embryonic chick heart are even smaller in diameter than those of the adult chicken, finch, and hummingbird hearts, and hence the average size of nexuses might also be expected to be smaller, this fact cannot explain the virtual absence of nexuses in the embryonic chick heart and frog heart. One is driven to presume either that, below the size seen in the adult finch and chicken, nexuses are not preserved or are not detectable, or that forms of close cell apposition other than the nexus

FIgURE 8 Hummingbird. The extended junctional SR (open arrow) at higher magnification shows the cribriform pattern. Note the circular profile (long arrow) apparently formed by a membrane of unit membrane configuration (small solid black arrow at top); that profile may represent a transverse section of a junctional process. Scale, $0.1 \mu . \times 186,000$.

Figure 9 Finch. Nuclear region $(N u c)$ of a fiber with dense granules is visible in Golgi cisternae (open arrow). Scale, $0.5 \mu . \times 28,500$.

FIgURE 10 Hummingbird. Similar dense granules are sometimes seen within the lumen of SR tubules (open arrow). Extracellular space, E. Scale, $0.25 \mu . \times 52,000$.

Figure 11 Dense granules, finch. Compare the similarity of the dense granule at the dark arrow with the one seen in the nerve cell $(N)$ at the open arrow. Extracellular space, $E$. Scale, $0.25 \mu . \times 41,500$. 


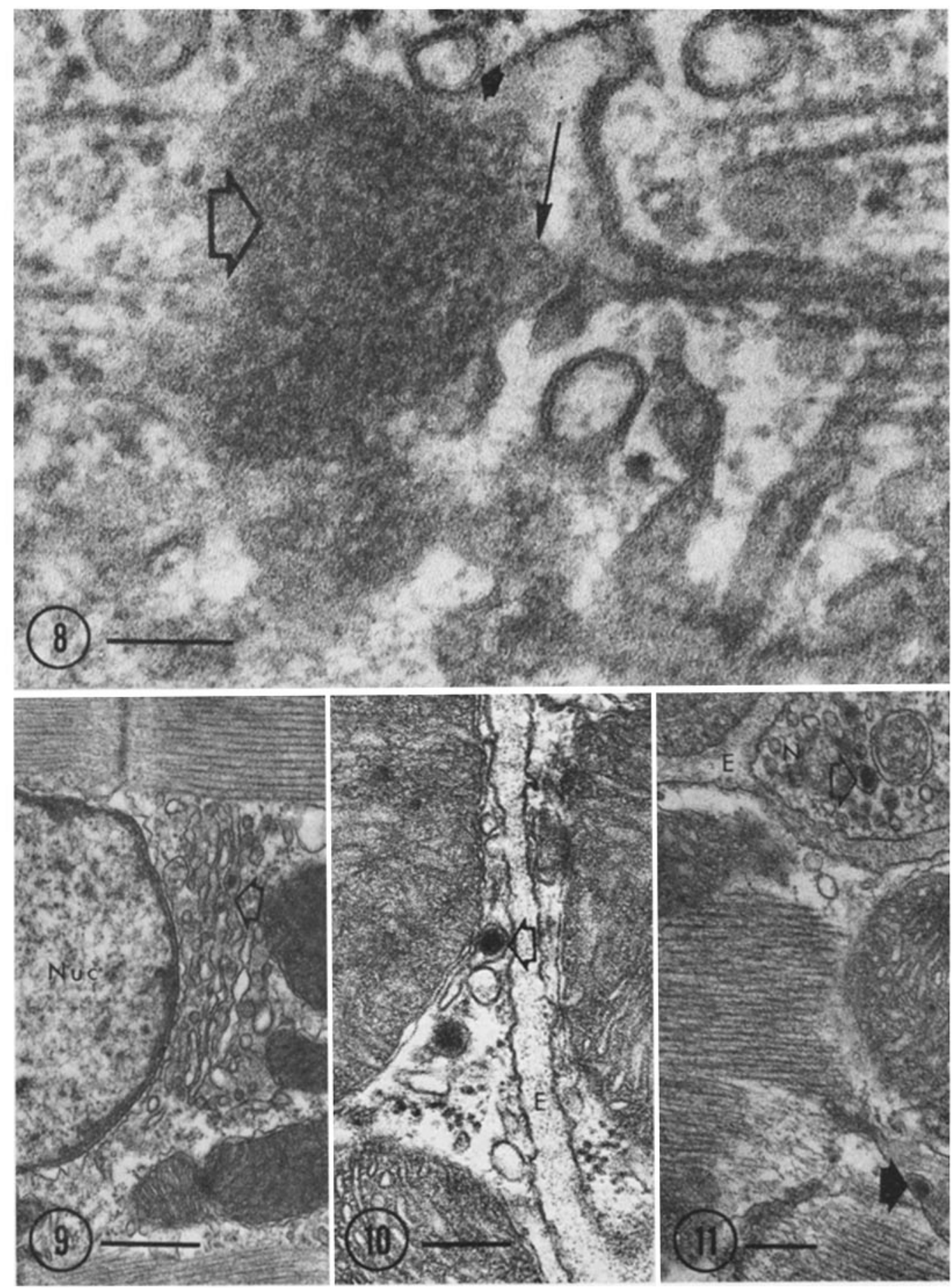

Paul H. Jewett, J. R. Sommer, and E. A. Johnson Cardiac Muscle 
form low resistance pathways between adjacent cells in these hearts (1).

\section{Sarcoplasmic Reticulum}

The discussion of the peculiar morphology of the sarcoplasmic reticulum of the hummingbird and finch will be facilitated by briefly recounting the structural components of the couplings $(10-12)$. The couplings are morphologically well-defined entities that occur in skeletal muscle and, mutatis mutandis, in cardiac muscle of many animals (Fig. 20 , schematic drawing). In cardiac muscle they are present as both interior and peripheral couplings. One of the invariant components of the couplings is the junctional SR, the limits of which are defined by the extent of the junctional granules which it contains, and by the extent of the junctional processes which emanate from the junctional SR toward, and make contact of some sort with, the sarcolemma. Although they are an integral part of the morphologic definition of the junctional SR and, thus, of the couplings, the junctional granules ("central membrane" of reference 10) have occasionally been observed in SR tubules that were not in contact with sarcolemma, and some of which were direct extensions of the junctional SR of couplings $(13,31)$. Such extensions of junctional SR, i.e. extensions having no sarcolemmal contact, are invariant components of the cardiac muscle fibers of hummingbirds and finches; this extended junctional SR envelops the $\mathrm{Z}$-line regions as a belt and is continuous with both, the many peripheral couplings on the one hand, and the remainder of the free $S R$ on the other.

The fine structure of the junctional $S R$ in skeletal and cardiac muscle has been studied in some detail $(21,22,32-36)$. In the case of cardiac muscle, the granular material inside the junctional SR has often a quasi-membranous appearance $(37,38)$ displaying periodic septa (Fig. 5 and reference 35 ). We have previously referred to this membranous structure as the "central membrane" (10), a designation which we later abandoned in favor of the term "junctional granules" (11) so as to tentatively simplify terminology when comparing these presumably homologous structures in different kinds of striated muscle; in skeletal muscle the granular material within junctional SR does not appear in the form of a quasi membrane.

What we refer to as junctional processes (11) have recently been the subject of special inquiry in skeletal muscle $(21,22,39)$, and the findings suggest that the structures in question are, indeed, processes originating from the envelope of the junctional SR. Looking at numerous couplings in the cardiac muscles of several animals, we find that the periodic densities seen between junctional $\mathrm{SR}$ and the sarcolemma appear in several forms depending on the various levels and angles at which the processes happen to be cut (12). The profiles thus obtained are not in conflict with the notion, expressed earlier $(10,40)$, that these processes are, perhaps, hollow tubules or cones. The formation of junctional processes may be the result of pull exerted on the SR membranes through the sites of attachment of the junctional SR to the sarcolemma (junctional SR of couplings), or to Z-line material (extended junctional SR). This notion is supported by the fact that junctional processes in couplings are often prominent only on

Figure 12 Adult hen. Ventricular fibers demonstrating small longitudinally oriented nexuses (arrow heads) within the intercalated disc are visible. Desmosome, $D$. Scale, $0.5 \mu . \times 29,000$.

Frgure 13 Finch. Typical nexus (cf. Figs. 14 and 15) showing a central gap (arrow). Scale, $0.05 \mu$. $\times 192,000$.

FIGURE 14. Hummingbird. An intercalated dise in longitudinal section reveals a nexus (open arrows) and desmosomes $(D)$. Note the network of free SR $(S R)$ cut close to the extracellular space. The meshes of this network often contain sarcolemmal vesicles $(P V)$. Extracellular space, $E$. Scale, $0.25 \mu . \times 48,000$.

Figure 15 Hummingbird. Note numerous nexuses (arrows) in the transverse section of the intercalated disc. A pocket of extracellular space with a peripheral coupling $(C)$ is visible at the arrow head; couplings do not occur at the junctional complexes that form the intercalated disc proper. Extended junctional SR continuous with a coupling is seen at the small solid black arrow at bottom. Sarcolemmal vesicle, $P V$; desmosome $D$; extracellular space, $E$. Scale, $0.25 \mu . \times 54,000$. 

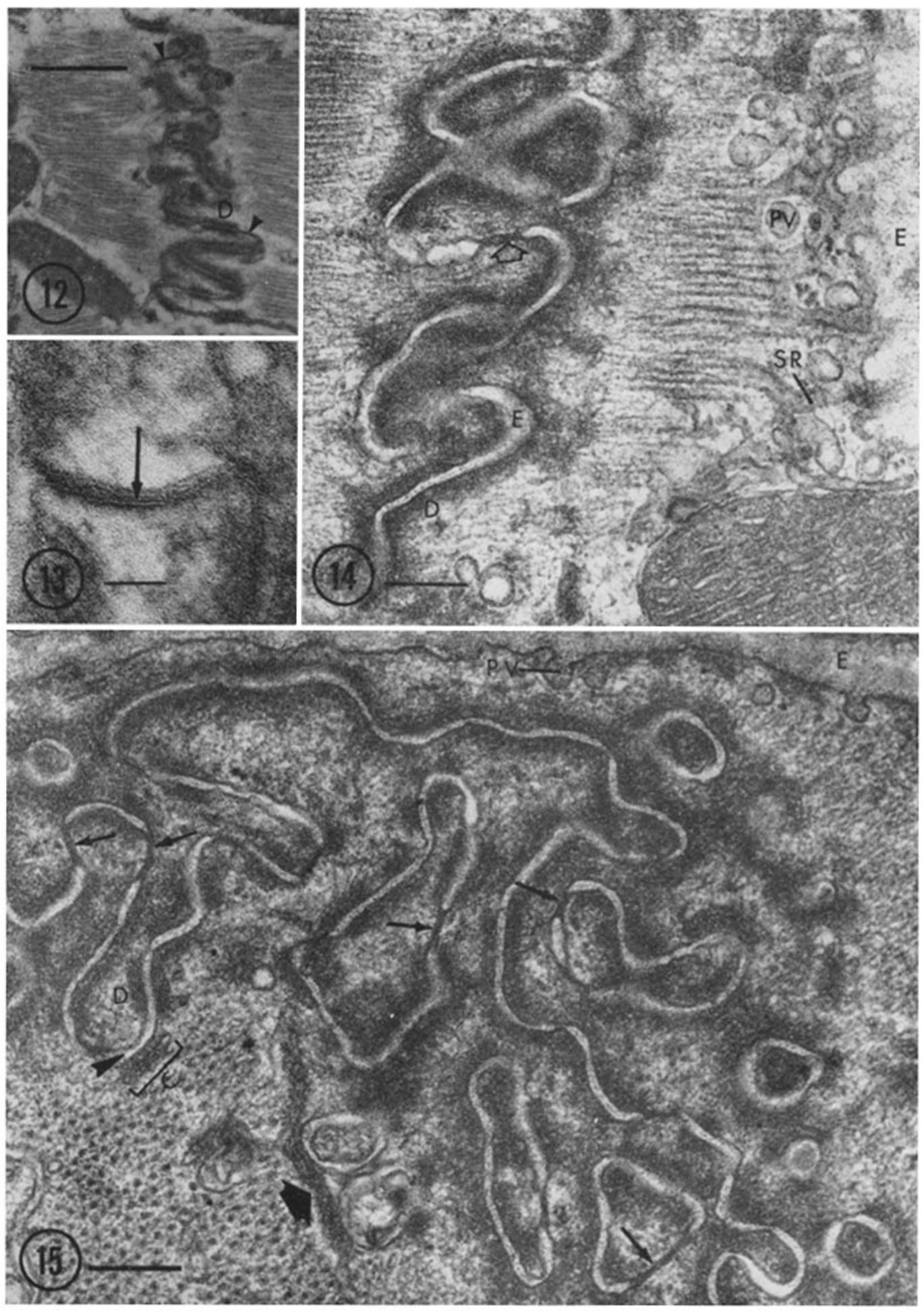

Paut, H. Jewett, J. R. Sommer, ano E. A. Johnson Cardiac Muscle 

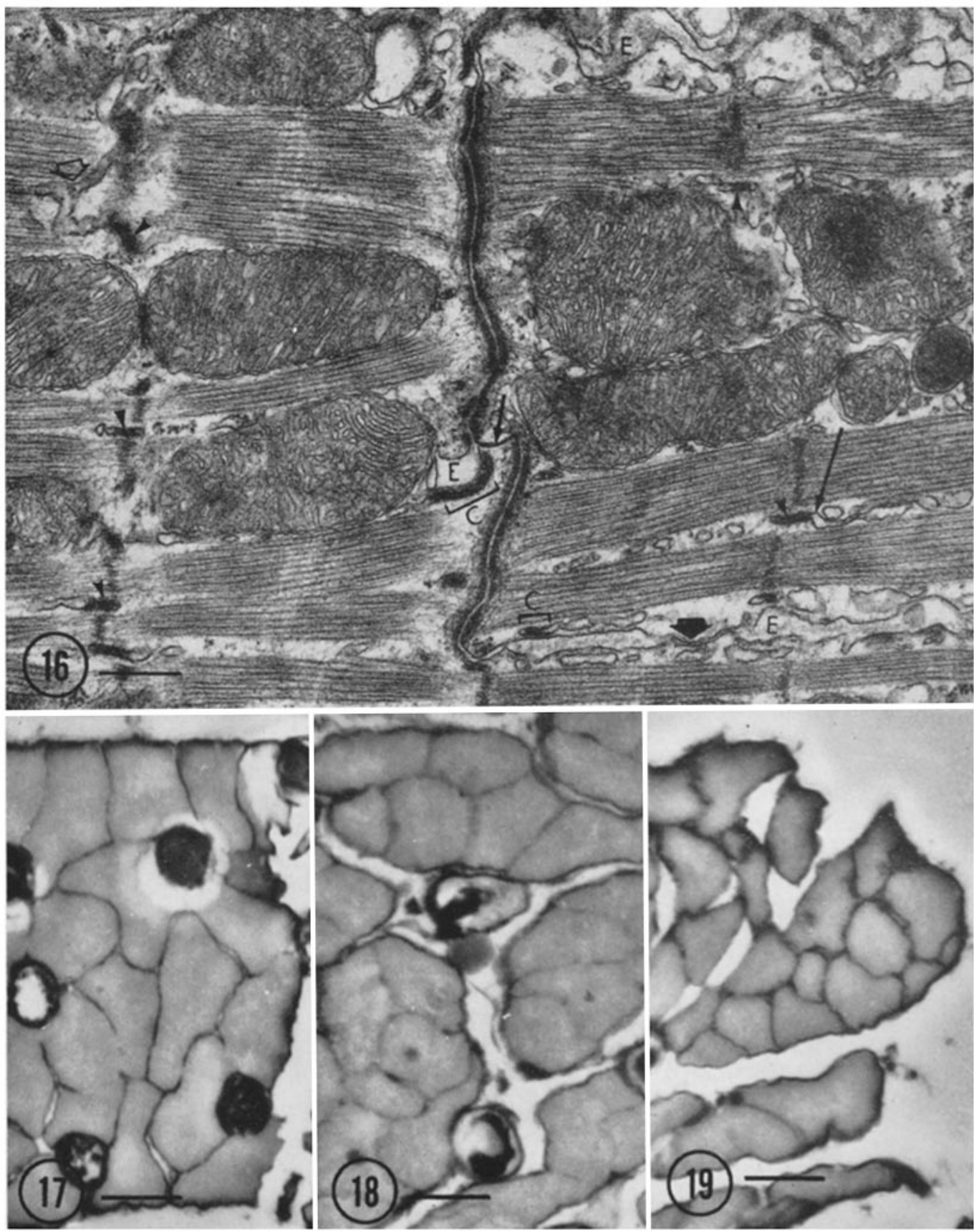

Figure 16 Finch. Occasionally, relatively straight intercalated dises are observed. A nexus is shown at the short arrow. Extended junctional SR, continuous with free SR (open arrow), is visible at long arrow. Note numerous peripheral couplings $(C)$ and profiles of extended junctional SR (arrowheads) Very close cell apposition at the small solid black arrow at hottom is evident. Extracellular space, $E$. Scale, $0.5 \mu . \times 23,000$.

Figure 17 Finch, ventricular fibers. Cell borders are outlined by exogenous peroxidase which fills the extracellular space. Mean filher diameter is $6.9 \mu$. Scale, $5 \mu$. $\times 2200$.

Figure 18 Adult hen, ventricular fibers in a similar preparation. Mean fiber diameter is 6.1 $\mu$. Scale, $5 \mu . \times 2200$

Figure 193 day old chick, ventricular fibers. Mean fiber diameter is $4.3 \mu$. Scale, $5 \mu . \times 2200$. 

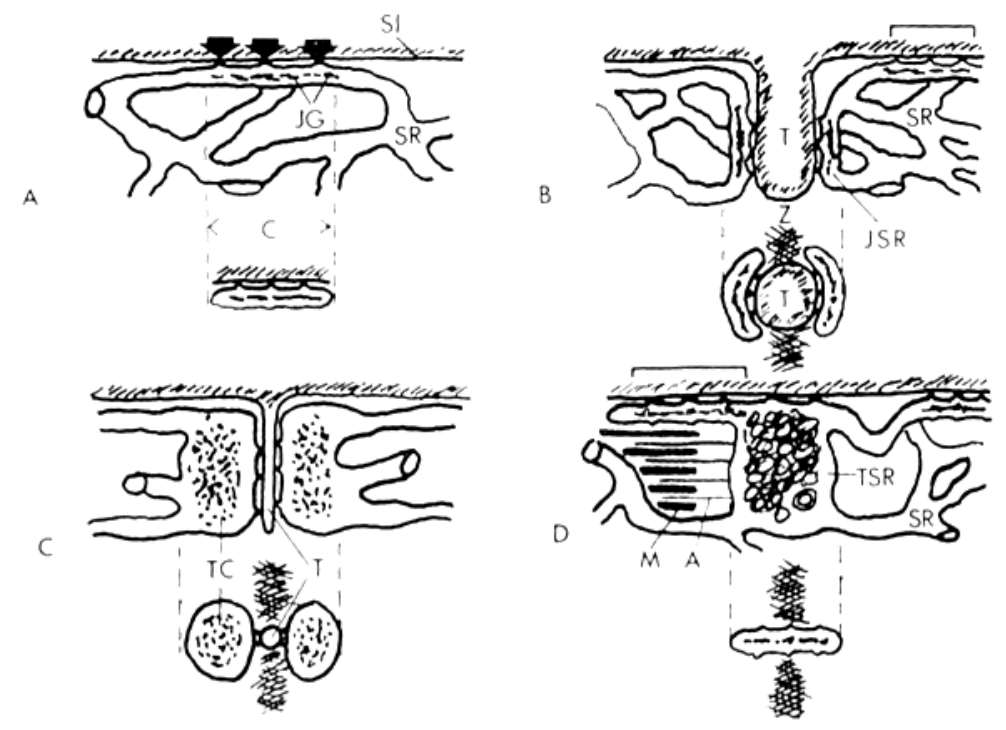

(20)

Figure 20 Schematic drawing of different kinds of couplings. A, B, and D, represent cardiac muscle; C represents a skeletal muscle. A. Peripheral coupling, sectioned in two planes perpendicular to one another. The extent of the coupling $(C)$ is defined by the extent of the junctional granules $(J G$, earlier called central membrane, see reference 10) within the junctional SR (SR), and the extent of the junctional processes (plump arrows) making contact with the sarcolemma $(S L)$. B. Interior coupling, two at a transverse tubule $(T)$, and one peripheral coupling (bracket) at the peripheral sarcolemma surrounding the entire cell. The former have the general configuration of the so-called triad of skeletal muscle (Fig. $20 \mathrm{C}$ ), i.e., two couplings facing each other across a transverse tubule; one coupling at a transverse tubule corresponds to a so-called dyad. The SR $(S R)$ is freely continuous with the SR on the other side of the transverse tubule, i.e. (indicated by two tubules disappearing behind transverse tubule) with the SR of adjacent sarcomeres within the cell. Junctional SR, JSR;Z line, Z. C. Interior coupling, two at a transverse tubule $(T)$ of a skeletal muscle (in this case at a $Z$ line; in other skeletal muscles, the transverse tubules are at the A-I junction). In skeletal muscle the junctional SR is usually referred to as terminal cisterna $(T C)$; it is dilated, has rare connections with the opposite junctional and free SR, and contains junctional granules. D. Extended junctional SR (TSR). It obscures the $Z$ line in this view and it is continuous with junctional SR of peripheral couplings (bracket). The electron-opaque material revealed in grazing sections through the extended junctional SR has a cribriform appearance which presumably reflects projections of superpositions of parts or all of the images produced by the junctional granules, the junctional processes, and the membranes forming the extended junctional SR. The junctional processes are always oriented toward $Z$ substance, toward sarcolemma, or both. Myosin, $M$, and actin, $A$, filaments are visible.

that side where the junctional SR faces and attaches to sarcolemma, but not where it faces free cytoplasm. On the other hand, the extended junctional SR, which lies between and attaches to Z-line material on both sides, carries junctional processes on both sides. The septa within the extended junctional SR (Fig. 5) may be related to maintaining the remarkably constant width of this specialized part of the SR. Cross-sections through the junctional processes of skeletal muscle, as
Kelly has suggested, reveal circular membranous profiles of roughly unit-membrane configuration (22). Similar profiles are seen in grazing sections cut through the extended junctional SR (Fig. 8). In Kelly's case, the junctional SR (terminal cisternae of skeletal muscle) is wide enough so that serial sections can just barely be informative as to the location of substructures. Such is not possible in cardiac muscle because of the small width of its junctional SR (approximately 25-35 $\mathrm{m} \mu$ ), even 
given very thin sections between, say, 10 and 20 $\mathrm{m} \mu$. Thus, in the case of cardiac muscle, virtually all structures belonging to the extended junctional SR, or junctional SR proper, will appear as superimposed projections. Images such as those seen in Figs. 7 and 8 (approximately $60-80-\mathrm{m} \mu$ thick sections) surely represent a moiré of structural patterns superimposed upon each other. The structures that possibly participate in the formation of the moiré seen in Fig. 8 are the unit membranes forming the extended junctional SR with its junctional processes, the junctional granules (central membrane), the material between the junctional granules and the unit membranes, and, finally, whatever tissue components outside the extended junctional SR may have been included in that section. By comparing Kelly's and our own observations, and by considering the special anatomic circumstances in each case (skeletal vs. cardiac muscle), we think it likely that circular profiles such as are seen in Fig. 8 represent crosssections through tubular or cone-shaped extensions (junctional processes) of the membranes of the extended junctional SR; for, in the case of the extended junctional SR, the sarcolemma is excluded, by definition, from contributing to the formation of such circular images (Fig. 8), and, in the case of skeletal muscle, the serial sections of Kelly (22) have at least minimized the possibility of such a contribution by the junctional granules. That the junctional processes are, in fact, processes the lumens of which are continuous with the lumen of the junctional SR has found support in the following observations. Quite fortuitously, we have filled with lanthanum some portions of the SR including the junctional SR of a few couplings of dog atrial muscle. The images thus obtained suggest that the junctional processes are filled together with, and from inside, the junctional SR (13).

The functional significance of junctional processes and, for that matter, of the coupling with all its components is suggested by experiments such as those of Huxley and Taylor (41) and, more recently, of Gage and Eisenberg (42), which show that transverse tubules and, presumably, the associated couplings are involved in the process of excitation-contraction coupling. The mechanisms by which excitation is coupled with the release of activator of contraction is obscure. Our own thoughts about the several possible mechanisms that come to mind are in enthusiastic agreement with Franzini-Armstrong's eloquent discussion of that problem (39).

The function of the extended junctional SR is unknown, but one point is worth considering. Extended junctional SR is absent in chicken hearts which beat at a much slower rate (up to 10 times) than the hearts of hummingbirds and finches (3, 43), and it is tempting to speculate that the presence or absence of extended junctional SR may be related to these differences in heart rates. Clearly, in rapidly beating hearts there is a need not only for rapid arrival at the myofilaments of released activator but also for rapid removal of it. Close anatomic association of the junctional SR with the sarcolemma would satisfy the role of coupling excitation with release of activator and, conceivably, with subsequent removal of it from the cell. But, the extension of the junctional SR into the cytoplasm shows that at least one of its functions is independent of the sarcolemma. The extended junctional SR could reflect the need for greater capacity, imposed by the greater heart rate, to remove the activator. However, assuming that the activator is released from the couplings, one still cannot exclude the possibility that the extended junctional SR also performs this function, in which case sarcolemmal depolarization, in some way, causes the release of activator from SR that is disconnected from the sarcolemma. Considering fast-beating mammalian hearts (43) in this context, it should be recalled that mammalian cardiac cells contain transverse tubules which form innumerable interior couplings along the $\mathrm{Z}$ lines; the junctional SR of these couplings can be viewed as the equivalent of the extended junctional SR occurring in bird cardiac cells which do not contain transverse tubules.

The apparent attachment of extended junctional $\mathrm{SR}$ to the $Z$ line was a consistent finding. Close association of a portion of SR (nonjunctional) with the $\mathrm{Z}$ line has been noted before (37), and recently electron-opaque strands have been described between the SR and Z-line material (44). The finding of similar linear densities between the extended junctional SR and the $Z$ line suggests physical attachment which may be especially significant, involving as it does, in this case, a highly specialized region of the $\mathrm{SR}$.

The tubules of the free SR (see Definitions, Criteria, and Dimensions), as well as some cisternae of the Golgi zone, occasionally, contain often globular electron-opaque material similar 
to that seen in chicken cardiac muscle (13). Distensions of the junctional SR which seem to be rather common in chicken cardiac muscle (1) have not been observed in the hearts of the hummingbird and finch. Indeed, a re-examination of chicken hearts may perhaps show that extended junctional SR does, in fact, exist in chicken cardiac muscle, albeit in a most rudimentary form.

Mr. Isaiah Taylor has again contributed his superior skill and judgement in all technical matters. Mrs. Maw has helped in the preparation of some of the material. We are most grateful to Dr. L. Lessin and Dr. G. Tisher for the permission to use their AEI-6B electron microscope

This investigation was supported in part by $\mathrm{Na}$ tional Institutes of Health Grant No. HE12486-01, and by NIH Grant No. 5-T01-HD00132.

Received for publication 7 July 1970, and in revised form 16 November 1970.

\section{REFERENCES}

1. Sommer, J. R., and E. A. Johnson. 1969. Z. Zellforsch. 98:437.

2. DiDio, L. 1967. Anat. Rec. 159:335.

3. Slautterback, D. B. 1965. J. Cell. Biol. 24:1.

4. Slautterback, D. B. 1966. In The Physiology and Biochemistry of Muscle as a Food. E. J. Briskey and J. C. Trautman, editors. University of Wisconsin Press, Madison, Wis.

5. Mizuhira, V., R. Hirakow, and H. Ozawa. 1967. In Electrophysiology and Ultrastructure of the Heart. T. Sano, V. Mizuhira, and K. Matsuda, editors. Grune and Stratton Inc., New York. 15.

6. Hama, K., and T. Kaneseki. 1967. In Electrophysiology and ultrastructure of the Heart. T. Sano, V. Mizuhira, and K. Matsuda, editors. Grune and Stratton Inc., New York. 27.

7. Hirakow, R. 1970. Amer. J. Cardiol. 25:195

8. Hirakow, R. 1966. Arch. Histol. Jap. $27: 485$.

9. Gossr Au, R. 1968. Histochemie. 13:111.

10. Johnson, E. A., and J. R. Sommer. 1967. J. Cell Biol. 33:103.

11. Sommer, J. R., and E. A. Johnson. 1968. J. Cell Biol. 36:497.

12. Sommer, J. R., and E. A. Johnson. 1970. Amer. J. Cardiol. 25:184.

13. Sommer, J. R., and P. H. Jewett. 1971. Cardiac muscle. A comparative ultrastructural anatomical view. In Myocardial Hypertrophy. Alpert, editor. Academic Press Inc., New York. 89.

14. Jewett, P. H., and J. R. Sommer. 1970. Fed. Proc. 29:390.
15. Dewey, M. M., and L. Barr. 1964. J. Cell. Biol. 23:553.

16. Revel, J. P., and M. J. Karnovsky. 1967. J. Cell Biol. 33:C7.

17. Truex, R. C., and M. Q. Smythe. 1965. Ann. N. Y. Acad. Sci. 127:19.

18. Karnovsky, M. J. 1965. J. Cell. Biol. 27:137A. (Abstr.)

19. Palade, G. E. 1952. J. Exp. Med. 95:285.

20. Graham, R. C., and M. J. Karnovsky. 1966. J. Histochem. Cytochem. 14:291.

21. Kelly, D. E. 1969. J. Ulirastruct. Res. 29:37.

22. Kelly, D. E., and W. A. Cahill. 1969. J. Cell Biol. 43(2, Pt. 2): 66 a. (Abstr.)

23. Sommer, J. R. 1968. J. Cell Biol. 39(2, Pt. 2): 127 A. (Abstr.)

24. Scotт, T. M. 1969. J. Anat. 106:173.

25. Brown, H. F., and S. G. Noble. 1969. J. Physiol. (London). 204:717.

26. Rougier, O., G. Vassort, D. Garnier, V. M. Gargouil, and E. Coraboeuf. 1969. Pfïgers Arch. Gesamte Physiol. Menschen Tiere. 308:91.

27. Weidmann, S. 1952. The electrical constants of Purkinje fibers. J. Physiol. (London). 118:348.

28. SAKamoto, T. 1969. Membrane characteristics of the canine papillary muscle fiber. J. Gen. Physiol. 54:765.

29. Sakamoto, Y., and M. Goto. 1970. A study of membrane constants in the dog myocardium. Jap. J. Physiol, 20:30.

30. Kamiyama, A., and K. Matiuda. 1966. Electrophysiological properties of the canine ventricular fiber. Jap. J. Physiol. 16:407.

31. Sommer, J. R. 1968. Fed. Proc. 27:357.

32. Walker, S. M., and G. R. Schrodt. 1966. Anat. Rec. 155:1.

33. Rosenbluth, J. 1969. J. Cell Biol. 42:817.

34. Revel, J. P. 1962. J. Cell. Biol. 12:571.

35. Walker, S. M., G. R. SGhrodt, and W. B. EDGe. 1970. Amrr. J. Anat. 128:33.

36. Simpson, F. O., and S. J. Oertelis. 1961. Nature. (London). 189:758.

37. Simpson, F. O., and D. G. Rayns. 1968. Amer. J. Anat. 122:193.

38. PAGE, E. 1966. Ultrastruct. Res. 17:72.

39. Franzini-Armstrong, C. 1970. J. Cell. Biol. 47: 488.

40. Birks, R. I. 1965. In Muscle. W. M. Paul, E. E. Daniel, C. M. Kay, and G. Monckton, editors. Pergamon Press Ltd., Oxford. 199.

41. Huxley, A. F., and R. E. TAYlor. 1958. J. Physiol. (London). 144:426.

42. Gage, P. W., and R. S. Eisengerg. 1967. Science (Washington). 158:1702.

43. Biological Data Book. 1964. P. L. Altman, and D. S. Dittwer. Federation of American Societies for Experimental Biology. 235.

44. Enge, M. B., and S. M. Walker. 1970. Anat. Rec. 166:51. 